\title{
NON-INVOLUTORIAL SPACE TRANSFORMATIONS ASSOCIATED WITH A LINEAR CONGRUENCE
}

\author{
M. L. VEST
}

1. Introduction. Vogt ${ }^{1}$ has studied, using synthetic methods, the space transformations associated with a linear congruence and with a bundle of lines. In the present paper the non-involutorial transformations associated with these configurations are found analytically, and by an entirely different geometric process. It will be shown that the general transformation associated with a linear congruence so obtained differs from that of Vogt in one important respect, having one more fundamental curve in each of the projectively related spaces. The transformations in the planes containing the directrices of the congruence are also shown.

Given the directrices $r$ and $s$ of the congruence and two projective pencils of surfaces

and

$$
\left|F_{m+n+1}\right|: r^{m} s^{n} g_{2 m+2 n+2 m n+1}
$$

$$
\left|F_{m^{\prime}+n^{\prime}+1}^{\prime}\right|: r^{m^{\prime}} s^{n^{\prime}} g_{2 m^{\prime}+2 n^{\prime}+2 m^{\prime} n^{\prime}+1} \text {. }
$$

Through a generic point $P(y)$ there passes a single $F$ of $|F|$. The unique line $t$ of the congruence through $P(y)$ meets the associated $F^{\prime}$ of $\left|F^{\prime}\right|$ in one residual point $P^{\prime}(x)$, the image of $P(y)$ under the transformation thus defined. The residual base curves of $|F|$ and $\left|F^{\prime}\right|$, other than $r$ and $s$, have been denoted by $g$ and $g^{\prime}$, respectively. Through a point $O_{g^{\prime}}$ on $g^{\prime}$ there is a unique line $t^{\prime}$ of the congruence, this line lying upon one surface of $\left|F^{\prime}\right|$. The associated surface of $|F|$ meets $t^{\prime}$ in a point $\bar{P}$ which generates a curve $\bar{g}$. Similarly, beginning with a point $O_{g}$ on $g$, a point $\bar{P}^{\prime}$ generating a curve $\bar{g}^{\prime}$ is found. It will be shown that $r, s, g, g^{\prime}, \bar{g}, \bar{g}^{\prime}$ are fundamental curves of the transformation.

2. Equations of the transformation. Let us take the equations of the directrices $r$ and $s$, respectively, as

$$
x_{1}=x_{2}=0, \quad x_{3}=x_{4}=0,
$$

and the pencils of surfaces

$$
\left|F_{m+n+1}\right| \equiv U-\lambda \bar{U}=0, \quad\left|F_{m^{\prime}+n^{\prime}+1}^{\prime}\right| \equiv U^{\prime}-\lambda \bar{U}^{\prime}=0
$$

Received by the editors January 24, 1942.

1 Vogt, Zentrale und windschiefe Raum-Verwandtschaften, Jahresbericht der Schlesischen Gesellschaft für Vaterlanddische Kultur, class 84, 1906, pp. 8-16. 
where

$$
\begin{aligned}
& U_{m+n+1}=\sum_{j, k=0}^{m, n} u_{j} v_{k}\left(c_{j k} x\right), \quad \bar{U}_{m+n+1}=\sum_{j, k=0}^{m, n} \bar{u}_{j} \bar{v}_{k}\left(\bar{c}_{j k} x\right), \\
& U_{m^{\prime}+n^{\prime}+1}^{\prime}=\sum_{j, k=0}^{m^{\prime}, n^{\prime}} u_{j}^{\prime} v_{k}^{\prime}\left(c_{j k}^{\prime} x\right), \quad \bar{U}_{m^{\prime}+n^{\prime}+1}^{\prime}=\sum_{j, k=0}^{m^{\prime}, n^{\prime}} \bar{u}_{j}^{\prime} \bar{v}_{k}^{\prime}\left(\bar{c}_{j k}^{\prime} x\right), \\
& u_{j}=\sum_{i=0}^{m} a_{i j} x_{1}^{i} x_{2}^{m-i}, \quad v_{k}=\sum_{i=0}^{n} b_{i k} x_{3}^{i} x_{4}^{n-i} \\
& \bar{u}_{j}=\sum_{i=0}^{m} \bar{a}_{i j} x_{1}^{i} x_{2}^{m-i}, \quad \quad \bar{v}_{k}=\sum_{i=0}^{n} \bar{b}_{i k} x_{3}^{i} x_{4}^{n-i} \\
& u_{j}^{\prime}=\sum_{i=0}^{m^{\prime}} a_{i j}^{\prime} x_{1}^{i} x_{2}^{m^{\prime}-i}, \quad v_{k}^{\prime}=\sum_{i=0}^{n^{\prime}} b_{i k}^{\prime} x_{3}^{i} x_{4}^{n^{\prime}-i}, \\
& \bar{u}_{j}^{\prime}=\sum_{i=0}^{m^{\prime}} \bar{a}_{i j}^{\prime} x_{1}^{i} x_{2}^{m^{\prime}-i}, \quad \quad \bar{v}_{k}^{\prime}=\sum_{i=0}^{n^{\prime}}{\bar{b}_{i k}}_{i}^{i} x_{3}^{i x_{4}^{\prime}-i}, \\
& \left(c_{j k} x\right)=c_{j k, 1} x_{1}+c_{j k, 2} x_{2}+c_{j k, 3} x_{3}+c_{j k, 4} x_{4},
\end{aligned}
$$

and so on.

Through a generic point $P(y)$ there is an $F$ of $|F|$ with parameter $\lambda=U(y) / \bar{U}(y)$ and to this corresponds the $F^{\prime}$ having equation

$$
U^{\prime}(x) \bar{U}(y)-\bar{U}^{\prime}(x) U(y)=0 .
$$

The unique line $t$ of the congruence passing through $P(y)$ intersects the surface (4) in the point $P^{\prime}$ having coordinates

where

$$
T_{m+m^{\prime}+n+n^{\prime}+3}^{-1}:\left\{\begin{array}{l}
\sigma x_{1}=R y_{1}+K y_{1}=S y_{1} \\
\sigma x_{2}=R y_{2}+K y_{2}=S y_{2} \\
\sigma x_{3}=R y_{3} \\
\sigma x_{4}=R y_{4}
\end{array}\right.
$$

$$
\begin{array}{rlrl}
K_{m+m^{\prime}+n+n^{\prime}+2} & =U^{\prime} \bar{U}-U \bar{U}^{\prime}, & R_{m+m^{\prime}+n+n^{\prime}+2} & =U \bar{W}^{\prime}-\bar{U} W^{\prime}, \\
W_{m^{\prime}+n^{\prime}+1}^{\prime} & =\sum_{j, k=0}^{m^{\prime}, n^{\prime}} u_{j}^{\prime} v_{k}^{\prime}\left(c_{j k}^{\prime} z\right), & \bar{W}_{m^{\prime}+n^{\prime}+1}^{\prime} & =\sum_{j, k=0}^{m^{\prime}, n^{\prime}} \bar{u}_{j}^{\prime} \bar{v}_{k}^{\prime}\left(\bar{c}_{j k}^{\prime} z\right), \\
\left(c_{j k} z\right) & =c_{j k, 1} y_{1}+c_{j k, 2} y_{2} .
\end{array}
$$

Equations (5) are those of the inverse transformation.

In a similar manner the equations of the direct transformation are found to be 
where

$$
T_{m+m^{\prime}+n+n^{\prime}+3}:\left\{\begin{array}{l}
\sigma y_{1}=R^{\prime} x_{1}+K^{\prime} x_{1}=S^{\prime} x_{1} \\
\sigma y_{2}=R^{\prime} x_{2}+K^{\prime} x_{2}=S^{\prime} x_{2} \\
\sigma y_{3}=R^{\prime} x_{3} \\
\sigma y_{4}=R^{\prime} x_{4}
\end{array}\right.
$$

$$
\begin{array}{rlrl}
K_{m+m^{\prime}+n+n^{\prime}+2}^{\prime} & =U \bar{U}^{\prime}-\bar{U} U^{\prime}=-K, R_{m+m^{\prime}+n+n^{\prime}+2}^{\prime} & =U^{\prime} \bar{W}-\bar{U}^{\prime} W, \\
W_{m+n+1} & =\sum_{j, k=0}^{m, n} u_{j} v_{k}\left(c_{j k} z\right), & \bar{W}_{m+n+1} & =\sum_{j, k=0}^{m, n} \bar{u}_{j} \bar{v}_{k}\left(\bar{c}_{j k} z\right) .
\end{array}
$$

3. Images of fundamental curves. The transformations $T^{-1}$ and $T$ applied to an $F^{\prime}$ and $F$ of $\left|F^{\prime}\right|$ and $|F|$, respectively, give

$$
U^{\prime} \sim\left(T^{-1}\right) U S^{m^{\prime}} R^{n^{\prime}} G, \quad U \sim(T) U^{\prime} S^{\prime m} R^{\prime n} G^{\prime}
$$

where

$$
\begin{aligned}
G_{2 m^{\prime}+2 n^{\prime}+2} & =U^{\prime} \bar{W}^{\prime}-\bar{U}^{\prime} W^{\prime}, \quad G_{2 m+2 n+2}^{\prime}=U \bar{W}-\bar{U} W, \\
S_{m+m^{\prime}+n+n^{\prime}+2} & =U \bar{M}^{\prime}-\bar{U} M^{\prime}, \quad S_{m+m^{\prime}+n+n^{\prime}+2}^{\prime}=U^{\prime} \bar{M}-\bar{U}^{\prime} M, \\
M_{m^{\prime}+n^{\prime}+1}^{\prime} & =W^{\prime}-U^{\prime}=\sum_{j, k=0}^{m^{\prime}, n^{\prime}} u_{j}^{\prime} v_{k}^{\prime}\left(c_{j k}^{\prime} w\right), \\
(10) & M_{m+n+1}=W-U=\sum_{j, k=0}^{m, n} u_{j} v_{k}\left(c_{j k} w\right), \\
\bar{M}_{m^{\prime}+n^{\prime}+1}^{\prime} & =\bar{W}^{\prime}-\bar{U}^{\prime}=\sum_{j, k=0}^{m^{\prime}, n^{\prime}} \bar{u}_{j}^{\prime} \bar{v}_{k}^{\prime}\left(\bar{c}_{j k}^{\prime} w\right), \\
\bar{M}_{m+n+1}=\bar{W}-\bar{U}=\sum_{j, k=0}^{m, n} \bar{u}_{j} \bar{v}_{k}\left(\bar{c}_{j k} w\right), &
\end{aligned}
$$

Here $U$ and $U^{\prime}$ are the corresponding surfaces of $|F|$ and $\left|F^{\prime}\right|$ and $g^{\prime} \sim\left(T^{-1}\right) G, s^{\prime} \sim\left(T^{-1}\right) S, g \sim(T) G^{\prime}, s \sim(T) S^{\prime}$. Similarly,

$$
\begin{aligned}
& K^{\prime} \sim\left(T^{-1}\right) K^{\prime} R^{n+n^{\prime}} S^{m+m^{\prime}} G G^{\prime}, \quad K \sim(T) K R^{\prime n+n^{\prime}} S^{\prime} m+m^{\prime} G G^{\prime}, \\
& K^{\prime} \sim(T) K^{\prime} R^{\prime n+n^{\prime}} S^{\prime m+m^{\prime}} G G^{\prime}, \quad K \sim(T) K R^{n+n^{\prime}} S^{m+m^{\prime}} G G^{\prime}, \\
& G^{\prime} \sim\left(T^{-1}\right) S^{2 m+1} R^{2 n+1} G^{\prime}, \quad G \sim(T) S^{\prime 2 m^{\prime}+1} R^{\prime 2 n^{\prime}+1} G, \\
& G^{\prime} \sim(T) S^{\prime 2 m+1} R^{\prime 2 n+1} G^{\prime}, \quad G \sim\left(T^{-1}\right) S^{2 m^{\prime}+1} R^{2 n^{\prime}+1} G, \\
& R^{\prime} \sim\left(T^{-1}\right) S^{m^{\prime}+m+1} R^{n^{\prime}+n} G G^{\prime}, \quad R \sim(T) S^{\prime m+m^{\prime}+1} R^{\prime n+n^{\prime}} G G^{\prime}, \\
& R^{\prime} \sim(T) S^{\prime m+m^{\prime}+1} R^{\prime n+n^{\prime}}\left[R^{\prime 2}-K^{\prime}\left(W^{\prime} \bar{W}-\bar{W}^{\prime} W\right)\right] \text {, } \\
& R \sim\left(T^{-1}\right) S^{m+m^{\prime}+1} R^{n+n^{\prime}}\left[R^{2}-K\left(W^{\prime} \bar{W}-\bar{W}^{\prime} W\right)\right], \\
& S^{\prime} \sim\left(T^{-1}\right) S^{m+m^{\prime}} R^{n+n^{\prime}+1} G G^{\prime}, \quad S \sim(T) S^{\prime m+m^{\prime}} R^{\prime n+n^{\prime}+1} G G^{\prime}, \\
& S^{\prime} \sim(T) S^{\prime m+m^{\prime}} R^{\prime n+n^{\prime}+1}\left[R^{\prime} S^{\prime}+K^{\prime}\left(W^{\prime} \bar{M}-\bar{W}^{\prime} M\right)\right], \\
& S \sim\left(T^{-1}\right) S^{m+m^{\prime}} R^{n+n^{\prime}+1}\left[R S+K\left(W \bar{M}^{\prime}-\bar{W} M^{\prime}\right)\right] .
\end{aligned}
$$


Through a point $O_{r}$ on $r$ there is a pencil of transversals through $s$. Each direction in this pencil determines an $F^{\prime}$ and the associated $F$ cuts the line in one residual point. The locus of such points is a plane curve $c$ which generates the surface $R$, image of $r$. The order of $c$, determined by the intersection of $R$ and a homaloidal surface, is $n+n^{\prime}+1$. Similarly, taking a point $O_{s}$ on $s$, we find a plane curve $k$ of order $m+m^{\prime}+1$ generating the surface $S$, image of $s$.

Through a point $O_{g^{\prime}}$ on $g^{\prime}$ there is a unique line $t$ of the congruence, but every $F$ of $|F|$ is associated with $O_{g^{\prime}}$, hence $O_{g^{\prime}} \sim\left(T^{-1}\right) t$. The ruled surface $G$ generated by $t$ is the image of $g^{\prime}$ under $T^{-1}$. Furthermore, every point $P^{\prime}$ of the transversal determines the same $F^{\prime}$ and $t$ meets the associated $F$ in one point $\bar{P}$ so that $\bar{P} \sim(T) t$. The locus of points $\bar{P}$ is the curve $\bar{g}$ and $\bar{g} \sim(T) G$. The order of $\bar{g}$, determined by the intersection of two homaloidal surfaces, is $2 m^{\prime} n^{\prime}+2 m n^{\prime}+2 m^{\prime} n+m$ $+3 m^{\prime}+n+3 n^{\prime}+3$. In a similar manner we find a curve $\bar{g}^{\prime}$ of order $2 m n+2 m n^{\prime}+2 m^{\prime} n+3 m+m^{\prime}+3 n+n^{\prime}+3$ such that $\bar{g}^{\prime} \sim\left(T^{-1}\right) G^{\prime}$.

We can now write the following correspondences:

$$
\begin{array}{rlrl}
r & \sim(T) R^{\prime}: s^{m+m^{\prime}+1} r^{n+n^{\prime}} g^{\prime} \bar{g}^{\prime}, & r \sim\left(T^{-1}\right) R: s^{m+m^{\prime}+1} r^{n+n^{\prime}} g \bar{g}, \\
s \sim(T) S^{\prime}: s^{m+m^{\prime}} r^{n+n^{\prime}+1} g^{\prime} \bar{g}^{\prime}, & & s \sim\left(T^{-1}\right) S: s^{m+m^{\prime}} r^{n+n^{\prime}+1} g \bar{g}, \\
g & \sim(T) G^{\prime}: s^{2 m+1} r^{2 n+1} g \bar{g}^{\prime}, & & g^{\prime} \sim\left(T^{-1}\right) G: s^{2 m^{\prime}+1} r^{2 n^{\prime}+1} \bar{g} g^{\prime}, \\
\bar{g}^{\prime} \sim\left(T^{-1}\right) G^{\prime}: s^{2 m+1} r^{2 n+1} g \bar{g}^{\prime}, & & \bar{g} \sim(T) G: s^{2 m^{\prime}+1} r^{2 n^{\prime}+1} \bar{g} g^{\prime} .
\end{array}
$$

4. Invariant and homaloidal surfaces. The eliminant of the paramter from $|F|$ and $\left|F^{\prime}\right|$ is the pointwise invariant surface $K$. Generic planes subjected to the transformations give

$$
\begin{aligned}
\pi^{\prime} & \equiv\left(A^{\prime} x\right) \sim\left(T^{-1}\right) R\left(A^{\prime} y\right)+K\left(A^{\prime} z\right)=\phi_{m+m^{\prime}+n+n^{\prime}+3} \\
\pi & \equiv(A y) \sim(T) R^{\prime}(A x)+K^{\prime}(A z)=\phi_{m+m^{\prime}+n+n^{\prime}+3}^{\prime}
\end{aligned}
$$

where the $\phi$ 's are homaloidal surfaces of the transformation. Further,

$$
\begin{aligned}
\phi & \sim(T)\left(A^{\prime} x\right) S^{\prime m+m^{\prime}+1} R^{\prime n+n^{\prime}+1} G G^{\prime}, \\
\phi^{\prime} & \sim\left(T^{-1}\right)(A y) S^{m+m^{\prime}+1} R^{n+n^{\prime}+1} G G^{\prime},
\end{aligned}
$$

hence the homaloidal webs are

$$
\infty^{3}|\phi|: s^{m+m^{\prime}+1} r^{n+n^{\prime}+1} g \bar{g}, \quad \infty^{3}\left|\phi^{\prime}\right|: s^{m+m^{\prime}+1} r^{n+n^{\prime}+1} g^{\prime} \bar{g}^{\prime} .
$$

The intersection of two homaloidal surfaces gives the homaloidal net

$$
\begin{aligned}
H & \equiv[\phi \phi]: s^{m^{2}+m^{\prime 2}+2 m m^{\prime}+2 m+2 m^{\prime}+1} \gamma^{n^{2}+2 n n^{\prime}+n^{\prime 2}+2 n+2 n^{\prime}+1} g \bar{g} b_{m+m^{\prime}+n+n^{\prime}+3}, \\
H^{\prime} & \equiv\left[\phi^{\prime} \phi^{\prime}\right]: s^{m^{2}+2 m m^{\prime}+m^{\prime 2}+2 m+2 m^{\prime}+1} \gamma^{n^{2}+2 n n^{\prime}+n^{\prime 2}+2 n+2 n^{\prime}+1} g^{\prime} \bar{g}^{\prime} b^{\prime}{ }_{m+m^{\prime}+n+n^{\prime}+3} .
\end{aligned}
$$

We now write the additional correspondences: 


$$
\begin{aligned}
& \pi \sim(T) \phi^{\prime}: s^{m+m^{\prime}+1} \gamma^{n+n^{\prime}+1} g^{\prime} \bar{g}^{\prime}, \quad \pi \sim\left(T^{-1}\right) \phi: s^{m+m^{\prime}+1} r^{n+n^{\prime}+1} g \bar{g}, \\
& K \sim(T) K^{\prime}: s^{m+m^{\prime}} r^{n+n^{\prime}} g g^{\prime} \bar{g} \bar{g}^{\prime}, \quad K^{\prime} \sim\left(T^{-1}\right) K ; s^{m+m^{\prime}} r^{n+n^{\prime}} g g^{\prime} \bar{g} \bar{g}^{\prime} .
\end{aligned}
$$

The jacobian of the transformation is $J=R G G^{\prime} S$.

5. Tangency along $r$ and $s$. The projectivity $x_{1}=y_{1}, x_{2}=y_{2}, x_{3}=k y_{3}$, $x_{4}=y_{3}+y_{4}$ is applied to the fundamental surfaces of the transformation and an examination of the coefficients of the highest powers of $x_{3}$ shows $K$ and $S$ to have

$$
\begin{aligned}
D_{m+m^{\prime}} \equiv & \sum_{j, k=0}^{m^{\prime}, n^{\prime}} u_{j}^{\prime}\left(\sum_{i=0}^{n^{\prime}} b_{i k} k^{i}\right)\left(k c_{j k, 3}^{\prime}+c_{j k, 4}^{\prime}\right) \\
& \cdot \sum_{j, k=0}^{m, n} \bar{u}_{j}\left(\sum_{i=0}^{n} \bar{b}_{i k} k^{i}\right)\left(k \bar{c}_{j k, 3}^{\prime}+\bar{c}_{j k, 4}\right) \\
& -\sum_{j, k=0}^{m, n} u_{j}\left(\sum_{i=0}^{n} b_{i k} k^{i}\right)\left(k c_{j k, 3}+c_{j k, 4}\right) \\
& \cdot \sum_{j, k=0}^{m^{\prime}, n^{\prime}} \bar{u}_{j}^{\prime}\left(\sum_{i=0}^{n^{\prime}} \bar{b}_{i k}^{\prime} k^{i}\right)\left(k \bar{c}_{j k, 3}^{\prime}+\bar{c}_{j k, 4}^{\prime}\right)=0
\end{aligned}
$$

as common tangent surface along $r . D$ is also a common tangent surface to $K^{\prime}$ and $S^{\prime}$ along $r$. A similar process shows

$$
\begin{aligned}
E_{n+n^{\prime}} \equiv & \sum_{j, k=0}^{m^{\prime}, n^{\prime}} v_{k}^{\prime}\left(\sum_{i=0}^{m^{\prime}} a_{i j}^{\prime} k^{i}\right)\left(k c_{j k, 1}^{\prime}+c_{j k, 2}^{\prime}\right) \\
& \cdot \sum_{j, k=0}^{m, n} \bar{v}_{k}\left(\sum_{i=0}^{m} \bar{a}_{i j} k^{i}\right)\left(k \bar{c}_{j k, 1}+\bar{c}_{j k, 2}\right) \\
- & \sum_{j, k=0}^{m, n} v_{k}\left(\sum_{i=0}^{m} a_{i j} k^{i}\right)\left(k c_{j k, 1}+c_{j k, 2}\right) \\
& \cdot \sum_{j, k=0}^{m^{\prime}, n^{\prime}} \bar{v}_{k}^{\prime}\left(\sum_{i=0}^{m^{\prime}} \bar{a}_{i j}^{\prime} k^{i}\right)\left(k \bar{c}_{j k, 1}^{\prime}+\bar{c}_{j k, 2}^{\prime}\right)=0
\end{aligned}
$$

to be common tangent surface to $K, K^{\prime}, R$ and $R^{\prime}$ along $s$.

6. Intersection table. Referring to (12), (13), and $\$ 5$ we can now write the following intersection table.

$$
\begin{aligned}
& {\left[R^{\prime} S^{\prime}\right]: s^{m^{2}+m^{\prime 2}+2 m m^{\prime}+m+m^{\prime}} r^{n^{2}+n^{\prime 2}+2 n n^{\prime}+n+n^{\prime}} g^{\prime} \bar{g}^{\prime}} \\
& {\left[R^{\prime} G^{\prime}\right]: s^{2 m^{2}+2 m m^{\prime}+3 m+m^{\prime}+1} r^{2 n^{2}+2 n n^{\prime}+n^{\prime}+n \bar{g}^{\prime}} d_{1,1} \cdots d_{1,2 m n+2 n}} \\
& {\left[R^{\prime} \phi^{\prime}\right]: s^{m^{2}+m^{\prime 2}+2 m m^{\prime}+2 m+2 m^{\prime}+1} r^{n^{2}+n^{\prime 2}+2 n n^{\prime}+n+n^{\prime}} g^{\prime} \bar{g}^{\prime} c_{n+n^{\prime}+1}} \\
& {\left[R^{\prime} K^{\prime}\right]: s^{m^{2}+m^{\prime 2}+2 m m^{\prime}+m+m^{\prime}} r^{n^{\prime 2}+2 n n^{\prime}+n^{2}+\left(n+n^{\prime}\right) t} g^{\prime} \bar{g}^{\prime}}
\end{aligned}
$$


$\left[S^{\prime} G^{\prime}\right]: s^{2 m^{2}+2 m m^{\prime}+m+m^{\prime}} r^{2 n^{\prime}+2 n n^{2}+3 n+n^{\prime}+1} \bar{g}^{\prime} e_{1,1} \cdots e_{1,2 m n+2 m}$

$\left[S^{\prime} \phi^{\prime}\right]: s^{m^{2}+m^{\prime 2}+2 m m^{\prime}+m+m^{\prime}} r^{n^{2}+n^{\prime 2}+2 n n^{\prime}+2 n+2 n^{\prime}+1} g^{\prime} \bar{g}^{\prime} k_{m+m^{\prime}+1}$

$\left[S^{\prime} K^{\prime}\right]: s^{m^{2}+2 m m^{\prime}+m^{\prime 2}+\left(m+m^{\prime}\right) t} \boldsymbol{r}^{n^{2}+n^{\prime 2}+2 n n^{\prime}+n+n^{\prime}} g^{\prime} \bar{g}^{\prime}$

$\left[G^{\prime} \phi^{\prime}\right]: s^{2 m^{2}+2 m^{\prime} m+3 m+m^{\prime}+1} \boldsymbol{r}^{2 n^{2}+2 n n^{\prime}+3 n+n^{\prime}+1} \bar{g}^{\prime} l_{1,1} \cdots l_{1,2 m+2 m n+2 n+1}$

$\left[G^{\prime} K^{\prime}\right]: s^{2 m^{2}+m+2 m m^{\prime}+m^{\prime}} r^{2 n^{2}+n+2 n n^{\prime}+n^{\prime}} g \bar{g}^{\prime}$

$\left[\phi^{\prime} K^{\prime}\right]: s^{m^{2}+m^{\prime 2}+2 m m^{\prime}+m+m^{\prime}} \boldsymbol{r}^{n^{2}+n^{\prime 2}+2 n n^{\prime}+n+n^{\prime}} g^{\prime} \bar{g}^{\prime} C_{m+m^{\prime}+n+n^{\prime}+2}$.

7. The $T_{n+n^{\prime}+2}$ in a plane through $r$. A plane $\pi \equiv x_{1}=\delta x_{2}$ cuts the surfaces of $|F|$ and $\left|F^{\prime}\right|$ in residual pencils of curves

$$
\left|f_{n+1}\right| \equiv u-\mu \bar{u}=0, \quad\left|f_{n^{\prime}+1}^{\prime}\right| \equiv u^{\prime}-\mu \bar{u}^{\prime}=0
$$

where

$$
\begin{array}{rlrl}
u_{n+1} & =\sum_{j, k=0}^{m, n} \psi_{j} \omega_{k}\left(\alpha_{j k} x\right), & \bar{u}_{n+1} & =\sum_{j, k=0}^{m, n} \bar{\psi}_{j} \bar{\omega}_{k}\left(\bar{\alpha}_{j k} x\right), \\
u_{n^{\prime}+1}^{\prime} & =\sum_{j, k=0}^{m^{\prime}, n^{\prime}} \psi_{j}^{\prime} \omega_{k}^{\prime}\left(\alpha_{j k}^{\prime} x\right), & \bar{u}_{n^{\prime}+1}^{\prime} & =\sum_{j, k=0}^{m^{\prime}, n^{\prime}} \bar{\psi}_{j}^{\prime} \bar{\omega}_{k}^{\prime}\left(\bar{\alpha}_{j k}^{\prime} x\right), \\
\psi_{j} & =\sum_{i=0}^{m} a_{i j} \delta^{i}, \quad \bar{\psi}_{j}=\sum_{i=0}^{m} \bar{a}_{i j} \delta^{i}, \quad \psi_{j}^{\prime}=\sum_{i=0}^{m^{\prime}} a_{i j}^{\prime} \delta^{i}, \quad \bar{\psi}_{j}^{\prime}=\sum_{i=0}^{m^{\prime}} \bar{a}_{i j}^{\prime} \delta^{i}, \\
\omega_{k} & =\sum_{i=0}^{n} b_{i k} x_{3}^{i} x_{4}^{n-i}, \\
\omega_{k}^{\prime} & =\sum_{i=0}^{n^{\prime}} b_{i k}^{\prime} x_{3}^{i} x_{4}^{n^{\prime}-i}, & \bar{\omega}_{k} & =\sum_{i=0}^{n} \bar{b}_{i k} x_{3}^{i} x_{4}^{n-i}, \\
\left(\alpha_{j k} x\right) & =\left(\alpha_{j k, 1} \delta+\alpha_{j k, 2}\right) x_{2}+\alpha_{j k, 3} x_{3}+\alpha_{j k, 4} x_{4} .
\end{array}
$$

The second directrix $s$ cuts $\pi$ in the point $P:(\delta, 1,0,0)$ which is an $n$-fold point on the curves of $|f|$ and an $n^{\prime}$-fold point on the curves of $\left|f^{\prime}\right|$. Through a generic point $P(y)$ of $\pi$ passes an $f$ of $|f|$ having parameter $\mu=u(y) / \bar{u}(y)$ and to this corresponds the $f^{\prime}$ of $\left|f^{\prime}\right|$ having equation

$$
u^{\prime}(x) \bar{u}(y)-\bar{u}^{\prime}(x) u(y)=0 .
$$

The unique transversal $t$ through $P(y)$ and $P$ meets (16) in a point $P^{\prime}(x)$, image of $P(y)$ under the transformation. The inverse transformation is thus

$$
T_{n+n^{\prime}+2}^{-1}: x_{2}=\sigma y_{2}+\kappa, \quad x_{3}=\sigma y_{3}, \quad x_{4}=\sigma y_{4},
$$

where 


$$
\begin{aligned}
\kappa_{n+n^{\prime}+2} & =u^{\prime} \bar{u}-\bar{u}^{\prime} u & \sigma_{n+n^{\prime}+1} & =u \bar{w}^{\prime}-\bar{u} w^{\prime}, \\
w_{n^{\prime}}^{\prime} & =\sum_{j, k=0}^{m^{\prime}, n^{\prime}} \psi_{j}^{\prime} \omega_{k}^{\prime}\left(\alpha_{j k}^{\prime} z\right), & \bar{w}_{n^{\prime}}^{\prime} & =\sum_{j, k=0}^{m^{\prime}, n^{\prime}} \bar{\psi}_{j}^{\prime} \bar{\omega}_{k}^{\prime}\left(\bar{\alpha}_{j k}^{\prime} z\right), \\
\left(\alpha_{j k} z\right) & =\alpha_{j k, 1} \delta+\alpha_{j k, 2} & &
\end{aligned}
$$

while the direct transformation is

$$
T_{n+n^{\prime}+2}: y_{2}=\sigma^{\prime} x_{2}+\kappa^{\prime}, \quad y_{3}=\sigma^{\prime} x_{3}, \quad y_{4}=\sigma^{\prime} x_{4}
$$

where

$$
\begin{aligned}
\kappa^{\prime} & =-\kappa, & \sigma_{n+n^{\prime}+1}^{\prime} & =u^{\prime} \bar{w}-\bar{u}^{\prime} w \\
w_{n} & =\sum_{j, k=0}^{m, n} \psi_{j} \omega_{k}\left(\alpha_{j k} z\right), & \bar{w}_{n} & =\sum_{j, k=0}^{m, n} \bar{\psi}_{j} \bar{\omega}_{k}\left(\bar{\alpha}_{j k} z\right) .
\end{aligned}
$$

The curve $\kappa$ is pointwise invariant under the transformations.

The application of the transformation and its inverse gives

$$
u^{\prime}(x) \sim\left(T^{-1}\right) \sigma^{n^{\prime}} u \gamma, \quad u(y) \sim(T) \sigma^{\prime n} u^{\prime} \gamma^{\prime},
$$

where $\gamma_{2 n^{\prime}+1}=u^{\prime} \bar{w}^{\prime}-\bar{u}^{\prime} w^{\prime}, \gamma_{2 n+1}^{\prime}=u \bar{w}-\bar{u} w$, and, furthermore,

$$
\begin{aligned}
& \kappa^{\prime} \sim\left(T^{-1}\right) \kappa^{\prime} \sigma^{n+n^{\prime}} \gamma \gamma^{\prime}, \quad \kappa \sim(T) \kappa \sigma^{\prime n+n^{\prime}} \gamma \gamma^{\prime}, \\
& \kappa^{\prime} \sim(T) \kappa^{\prime} \sigma^{\prime n+n^{\prime}} \gamma \gamma^{\prime}, \quad \kappa \sim\left(T^{-1}\right) \kappa \sigma^{n+n^{\prime}} \gamma \gamma^{\prime}, \\
& \gamma^{\prime} \sim\left(T^{-1}\right) \sigma^{2 n+1} \gamma^{\prime}, \quad \gamma \sim(T) \sigma^{\prime 2 n^{\prime}+1} \gamma, \\
& \gamma^{\prime} \sim(T) \sigma^{2 n+1} \gamma^{\prime}, \quad \gamma \sim\left(T^{-1}\right) \cdot \sigma^{2 n^{\prime}+1} \gamma, \\
& \sigma^{\prime} \sim\left(T^{-1}\right) \sigma^{n+n^{\prime}} \gamma \gamma^{\prime}, \quad \sigma \sim(T) \sigma^{\prime n+n^{\prime}} \gamma \gamma^{\prime}, \\
& \sigma^{\prime} \sim(T) \sigma^{\prime n+n^{\prime}}\left[\sigma^{\prime 2}+\kappa^{\prime}\left(w^{\prime} \bar{w}-w \bar{w}^{\prime}\right)\right] \text {, } \\
& \sigma \sim\left(T^{-1}\right) \sigma^{n+n^{\prime}}\left[\sigma^{2}+\kappa\left(w^{\prime} \bar{w}-w \bar{w}^{\prime}\right)\right] .
\end{aligned}
$$

The base points of the pencil $|f|$ are $P$ and $2 n+1$ other points $\Gamma_{1}, \Gamma_{2}, \cdots, \Gamma_{2 n+1}$ while those of $\left|f^{\prime}\right|$ are $P$ and $\Gamma_{1}^{\prime}, \Gamma_{2}^{\prime}, \cdots, \Gamma_{2 n^{\prime}+1}^{\prime}$. We designate the lines $P \Gamma_{1}, P \Gamma_{2}, \cdots, P \Gamma_{2 n+1}$ as $\gamma_{1}^{\prime}, \gamma_{2}^{\prime}, \cdots, \gamma_{2 n+1}^{\prime}$ and $P \Gamma_{1}^{\prime}, P \Gamma_{2}^{\prime}, \cdots, P \Gamma_{2 n^{\prime}+1}^{\prime}$ as $\gamma_{1}, \gamma_{2}, \cdots, \gamma_{2 n^{\prime}+1}$. The curves $\gamma$ and $\gamma^{\prime}$ in (21) consist of the lines $\gamma_{1}, \gamma_{2}, \cdots, \gamma_{2 n^{\prime}+1}$ and $\gamma_{1}^{\prime}, \gamma_{2}^{\prime}, \cdots, \gamma_{2 n+1}^{\prime}$, respectively. It is easily seen that $\Gamma_{i} \sim(T) \gamma_{i}^{\prime}, \Gamma_{i}^{\prime} \sim\left(T^{-1}\right) \gamma_{i}, \gamma_{i}^{\prime} \sim\left(T^{-1}\right) \Gamma_{i}$, $\gamma_{i} \sim(T) \Gamma_{i}^{\prime}$.

Moreover, there is a particular $f$ of $|f|$ consisting of the lines $\gamma_{1}^{\prime}$ and an $n$-ic through $\Gamma_{2}, \Gamma_{3}, \cdots, \Gamma_{2 n+1}, P$, the latter being an $(n-1)$ fold point on the $n$-ic. The $n$-ic intersects $\gamma_{1}^{\prime}$ in another point $\bar{P}_{1}$. Then $\bar{P}_{1} \sim(T) \bar{\Gamma}_{1}^{\prime}$ and $\bar{\Gamma}_{1}^{\prime} \sim\left(T^{-1}\right) \gamma_{1}^{\prime}$. Similarly, we can find points $\bar{\Gamma}_{2}^{\prime}, \cdots, \bar{\Gamma}_{2 n+1}^{\prime}, \bar{\Gamma}_{1}, \cdots, \bar{\Gamma}_{2 n^{\prime}+1}$ such that $\bar{\Gamma}_{i}^{\prime} \sim\left(T^{-1}\right) \gamma_{i}^{\prime}$ and $\bar{\Gamma}_{i} \sim(T) \gamma_{i}$. 
We can now write the following correspondences:

$$
\begin{aligned}
P & \sim(T) \rho^{\prime}: P^{n+n^{\prime}} \Gamma_{1}^{\prime} \cdots \Gamma_{2 n^{\prime}+1}^{\prime} \bar{\Gamma}_{1}^{\prime} \cdots \bar{\Gamma}_{2 n+1}^{\prime}, \\
\Gamma_{i} & \sim(T) \gamma_{i}^{\prime}: P \Gamma_{i} \bar{\Gamma}_{i}^{\prime}, \\
\bar{\Gamma}_{i}^{\prime} & \sim\left(T T^{-1}\right) \gamma_{i}^{\prime}: P \Gamma_{i} \bar{\Gamma}_{i}^{\prime}, \\
\kappa & \sim(T) \kappa^{\prime}: P^{n+n^{\prime}} \Gamma_{1} \cdots \Gamma_{2 n+1} \Gamma_{1}^{\prime} \cdots \Gamma_{2 n^{\prime}+1}^{\prime} \bar{\Gamma}_{1} \cdots \bar{\Gamma}_{2 n^{\prime}+1} \bar{\Gamma}_{1}^{\prime} \cdots \bar{\Gamma}_{2 n+1}^{\prime} .
\end{aligned}
$$

When the transformations are applied to a generic line we get

$$
\begin{gathered}
\lambda^{\prime} \equiv(A x) \sim\left(T^{-1}\right) \sigma(A y)+\kappa A_{2}=\phi_{n+n^{\prime}+2}, \\
\lambda \equiv(A y) \sim(T) \sigma^{\prime}(A x)+\kappa^{\prime} A_{2}=\phi_{n+n^{\prime}+2}^{\prime}, \\
\phi \sim(T)(A y) \sigma^{\prime n+n^{\prime}+1} \gamma \gamma^{\prime}, \quad \phi^{\prime} \sim\left(T^{-1}\right)(A x) \sigma^{n+n^{\prime}+1} \gamma \gamma^{\prime}
\end{gathered}
$$

and the holaloidal nets are therefore

$$
\begin{gathered}
\infty^{2}|\phi|: P^{n+n^{\prime}+1} \Gamma_{1} \cdots \Gamma_{2 n+1} \bar{\Gamma}_{1} \cdots \bar{\Gamma}_{2 n^{\prime}+1}, \\
\infty^{2}\left|\phi^{\prime}\right|: P^{n+n^{\prime}+1} \Gamma_{1}^{\prime} \cdots \Gamma_{2 n^{\prime}+1}^{\prime} \bar{\Gamma}_{1}^{\prime} \cdots \bar{\Gamma}_{2 n+1}^{\prime} .
\end{gathered}
$$

The translation $x_{2}=x_{2}^{\prime}+1, x_{3}=x_{3}^{\prime}, x_{4}=x_{4}^{\prime}$ is applied to the fundamental curves of the transformation and $\sigma^{\prime}$ and $\kappa^{\prime}$ are found to have the curve

$$
\begin{aligned}
l_{n+n^{\prime}} \equiv & \sum_{j, k=0}^{m^{\prime}, n^{\prime}} \psi^{\prime} \omega^{\prime}\left(\alpha_{j k, 1}^{\prime} \delta+\alpha_{j k, 2}^{\prime}\right) \cdot \sum_{j, k=0}^{m, n} \bar{\psi} \bar{\omega}\left(\bar{\alpha}_{j k, 1} \delta+\bar{\alpha}_{j k, 2}\right) \\
& -\sum_{j, k=0}^{m^{\prime}, n^{\prime}} \bar{\psi}^{\prime} \bar{\omega}^{\prime}\left(\bar{\alpha}_{j k, 1}^{\prime} \delta+\bar{\alpha}_{j k, 2}^{\prime}\right) \cdot \sum_{j, k=0}^{m, n} \psi \omega\left(\alpha_{j k, 1} \delta+\alpha_{i k, 2}\right)=0
\end{aligned}
$$

as common tangent at the point $P$.

The jacobian is $\sigma^{2} \gamma \gamma^{\prime}$.

The intersection table is as follows

$$
\begin{aligned}
& \left\lfloor\phi^{\prime} \phi^{\prime}\right]: P^{n^{2}+2 n n^{\prime}+n^{\prime 2}+2 n+2 n^{\prime}+1} \mathrm{\Gamma}_{1}^{\prime} \ldots \mathrm{\Gamma}_{2 n^{\prime}+1}^{\prime} \bar{\Gamma}_{1}^{\prime} \ldots \bar{\Gamma}_{2 n+1}^{\prime} F \\
& \text { (the homaloidal web) } \\
& {\left[\sigma^{\prime} \gamma_{i}^{\prime}\right]: P^{n+n^{\prime}} \bar{\Gamma}_{i}^{\prime}, \quad\left[\gamma_{i}^{\prime} \kappa^{\prime}\right]: P^{n+n^{\prime}} \Gamma_{i} \bar{\Gamma}_{i}^{\prime}, \quad\left[\gamma_{i}^{\prime} \phi^{\prime}\right]: P^{n+n^{\prime}+1} \bar{\Gamma}_{i}^{\prime}} \\
& \left\lfloor\sigma^{\prime} \kappa^{\prime}\right]: P^{n^{2}+2 n n^{\prime}+n^{\prime 2}+\left(n+n^{\prime}\right) t} \Gamma_{1}^{\prime} \ldots \Gamma_{2 n^{\prime}+1}^{\prime} \bar{\Gamma}_{1}^{\prime} \ldots \bar{\Gamma}_{2 n+1}^{\prime} \\
& \left\lfloor\sigma^{\prime} \phi^{\prime}\right]: P^{n^{2}+2 n n^{\prime}+n^{\prime 2}+n+n^{\prime}} \Gamma_{1}^{\prime} \cdots \Gamma_{2 n^{\prime}+1}^{\prime} \bar{\Gamma}_{1}^{\prime} \cdots \bar{\Gamma}_{2 n+1}^{\prime} \\
& {\left[\kappa^{\prime} \phi^{\prime}\right]: P^{n^{2}+2 n n^{\prime}+n^{\prime 2}+n+n^{\prime}} \Gamma_{1}^{\prime} \cdots \Gamma_{2 n^{\prime}+1}^{\prime} \bar{\Gamma}_{1}^{\prime} \cdots \bar{\Gamma}_{2 n+1}^{\prime} \text {. }}
\end{aligned}
$$

As the plane $\pi$ generates the pencil on $r$ the $T_{n+n^{\prime}+2}$ generates the 
space $T_{m+m^{\prime}+n+n^{\prime}+3}$ whose equations may be obtained from (17) and (19) by replacing $u, \bar{u}, u^{\prime}, \bar{u}^{\prime}, w, \bar{w}, w^{\prime}, \bar{w}^{\prime},(\alpha z)$ and $\delta$ by $U, \bar{U}, U_{\urcorner}^{\prime}, \bar{U}^{\prime}$, $W, \bar{W}, W^{\prime}, \bar{W}^{\prime},(c z)$ and $x_{1} / x_{2}$, respectively.

When the surfaces are cut by a plane $\pi^{\prime} \equiv x_{3}=\epsilon x_{4}$ through $s$ a similar plane transformation of order $m+m^{\prime}+2$ is found.

8. The transformation associated with a special linear congruence. If we take the directrices $r$ and $s$ of the congruence as

$$
x_{1}=x_{4}=0, \quad x_{2}=x_{4}=0,
$$

respectively, and pencils of surfaces $F$ and $F^{\prime}$ as in (2) and (3) where $u_{j}=\sum_{i=0}^{m} a_{i j} x_{1}^{i} x_{4}^{m-i}, v_{k}=\sum_{i=0}^{n} b_{i k} x_{2}^{i} x_{4}^{n-i}$ and similarly for $\bar{u}_{j}, u_{j}^{\prime}$, and so on, the equations of the inverse transformation obtained by the present technique are

$$
T_{m+m^{\prime}+n+n^{\prime}+2}^{-1}: x_{1}=R y_{1}, \quad x_{2}=R y_{2}, \quad x_{3}=R y_{3}+K, \quad x_{4}=R y_{4}
$$

where $K_{m+m^{\prime}+n+n^{\prime}+2}=U^{\prime} \bar{U}-U \bar{U}^{\prime}, R_{m+m^{\prime}+n+n^{\prime}+1}=U \bar{W}^{\prime}-\bar{U} W^{\prime}$,

$$
W_{m^{\prime}+n^{\prime}}^{\prime}=\sum_{j, k=0}^{m^{\prime}, n^{\prime}} u_{j}^{\prime} v_{k}^{\prime}\left(c_{j k, 3}^{\prime}\right), \quad \bar{W}_{m^{\prime}+n^{\prime}}^{\prime}=\sum_{j, k=0}^{m^{\prime}, n^{\prime}} \bar{u}_{j}^{\prime} \bar{v}_{k}^{\prime}\left(\bar{c}_{j k, 3}^{\prime}\right) .
$$

Such transformations are well known so that no discussion will be given.

9. Discussion. Vogt, in his general skew transformation, finds three fundamental curves and their images in each space. These he designates as $g_{1}, g_{2}, l_{1}, l_{2}, R_{1}, R_{2}$ having images $\gamma_{2}, \gamma_{1}, \lambda_{2}, \lambda_{1}, \rho, \rho$, respectively. Of these $g_{1}, g_{2}, l_{1}, l_{2}, \gamma_{2}, \gamma_{1}, \lambda_{2}, \lambda_{1}$, correspond to $r, r, s, s, R^{\prime}, R$, $S^{\prime}, S$ of the present paper. However, $R_{1}, R_{2}, \rho, \rho$, are replaced by $g, \bar{g} ; g^{\prime}, \bar{g}^{\prime} ; G^{\prime}, G ; G, G^{\prime}$, respectively. The sums of the orders of $g$ and $\bar{g}, g^{\prime}$ and $\bar{g}^{\prime}, G$ and $G^{\prime}$ are equal to the orders of $R_{1}, R_{2}$, and $\rho$, respectively, as given by Vogt.

The properties of the transformation in $\$ 8$ are the same as those of the "central" transformation which he describes.

Quadratic transformations associated with a linear congruence may of course be gotten from those of the present paper by taking $|F|$ and $\left|F^{\prime}\right|$ as pencils of planes, that is, making $m=n=0$, in the plane transformations of $\$ 7$ and the space transformation of $\$ 8$.

West Virginia UNIVERSITY 